\title{
Real Power Loss Minimization and Voltage Stability Enhancement by Hybridization of Eagle Strategy with Particle Swarm Optimization Algorithm
}

\author{
K. Lenin \\ Department of EEE, Prasad V. Potluri Siddhartha Institute of Technology \\ Kanuru, Vijayawada, Andhra Pradesh - 520007. \\ Corresponding author: gklenin@gmail.com
}

Received: Sep 21, 2017

Revised: Dec 25, 2017

Accepted: Jan 12, 2018

\begin{abstract}
In this paper, Hybridization of Eagle Strategy (ES) with Particle Swarm Optimization is proposed to solve the optimal reactive power dispatch Problem. Proposed hybridization of Eagle Strategy with Particle Swarm Optimization (EPSO) enhances the search in rigorous mode. Eagle strategy has been instigating by the foraging behaviour of golden eagles. This stratagem has two important parameters: arbitrary search and exhaustive chase. At first it explores the search space globally, and then in the second case the strategy makes an intensive local search with using an effective local optimizer method. So, Particle Swarm Optimization has been enhanced using ES and employed to solve reactive power optimization problem. In order to appraise the efficiency of the projected EPSO algorithm, it has been tested in standard IEEE 30 bus system and compared other reported algorithms. Results show's that EPSO algorithm is more efficient in plummeting the real power loss and voltage index also enhanced.
\end{abstract}

Keywords: Optimal reactive power, transmission loss, eagle strategy, particle swarm optimization

\section{Introduction}

In recent years the optimal reactive power dispatch (ORPD) problem has received great attention as a result of the improvement on economy and security of power system operation. Solutions of ORPD problem aim to minimize object functions such as fuel cost, power system loses, etc. while satisfying a number of constraints like limits of bus voltages, tap settings of transformers, reactive and active power of power resources and transmission lines and a number of controllable Variables $[1,2]$. In the literature, many methods for solving the ORPD problem have been done up to now. At the beginning, several classical methods such as gradient based [12], interior point [11], linear programming [7] and quadratic programming [10] have been successfully used in 
order to solve the ORPD problem. However, these methods have some disadvantages in the Process of solving the complex ORPD problem. Drawbacks of these algorithms can be declared insecure convergence properties, long execution time, and algorithmic complexity. Besides, the solution can be trapped in local minima $[1,3]$. In order to overcome these disadvantages, researches have successfully applied evolutionary and heuristic algorithms such as Genetic Algorithm (GA) [2], Differential Evolution (DE) [4] and Particle Swarm Optimization (PSO) [13]. It is reported in those that evolutionary or heuristic algorithms are more efficient than classical algorithms for solving the reactive power problem. During the last decades a lot of population-based Meta heuristic algorithms were proposed. Voltage stability evaluation using modal analysis [6] is used as the indicator of voltage stability. In this paper hybridization of Eagle Strategy with Particle Swarm Optimization (EPSO) algorithm is proposed to solve the optimal reactive power dispatch Problem. Eagle strategy (ES) has been instigating by the foraging behaviour of golden eagles. This stratagem has two important parameters: arbitrary search and exhaustive chase. At first it explores the search space globally, and then in the second case the strategy makes an intensive local search with using an effective local optimizer method. So, Particle Swarm Optimization (PSO) has been enhanced using ES and employed to solve reactive power optimization problem. The performance of hybridized Eagle Strategy with Particle Swarm Optimization (EPSO) has been evaluated in standard IEEE 30 bus test system and the results analysis shows that our proposed approach outperforms all approaches investigated in this paper.

\section{Voltage Stability Evaluation}

\subsection{Modal Analysis for Voltage Stability Evaluation}

Modal analysis is one among best methods for voltage stability enhancement in power systems. The steady state system power flow equations are given by.

$$
\left[\begin{array}{l}
\Delta \mathrm{P} \\
\Delta \mathrm{Q}
\end{array}\right]=\left[\begin{array}{cc}
\mathrm{J}_{\mathrm{p} \theta} & \mathrm{J}_{\mathrm{pv}} \\
\mathrm{J}_{\mathrm{q} \theta} & \mathrm{J}_{\mathrm{QV}}
\end{array}\right]\left[\begin{array}{l}
\Delta \theta \\
\Delta V
\end{array}\right]
$$

where

$$
\begin{aligned}
& \Delta \mathrm{P}=\text { Incremental change in bus real power. } \\
& \Delta \mathrm{Q}=\text { Incremental change in bus reactive Power injection } \\
& \Delta \theta=\text { incremental change in bus voltage angle. } \\
& \Delta \mathrm{V}=\text { Incremental change in bus voltage Magnitude }
\end{aligned}
$$

$\mathrm{J}_{\mathrm{p} \theta}, \mathrm{J}_{\mathrm{pv}}, \mathrm{J}_{\mathrm{q} \theta}, \mathrm{J}_{\mathrm{QV}}$ are Jacobian matrices and the sub-matrixes of the System voltage stability. It affected by both $\mathrm{P}$ and $\mathrm{Q}$.

To reduce (1), let $\Delta \mathrm{P}=0$, then 
24 Real Power Loss Minimization and Voltage Stability Enhancement by Hybridization of Eagle Strategy ...

$$
\begin{gathered}
\Delta \mathrm{Q}=\left[\mathrm{J}_{\mathrm{QV}}-\mathrm{J}_{\mathrm{Q} \theta} \mathrm{J}_{\mathrm{P} \theta^{-1}} \mathrm{~J}_{\mathrm{PV}}\right] \Delta \mathrm{V}=\mathrm{J}_{\mathrm{R}} \Delta \mathrm{V} \\
\Delta \mathrm{V}=\mathrm{J}^{-1}-\Delta \mathrm{Q}
\end{gathered}
$$

where

$$
\mathrm{J}_{\mathrm{R}}=\left(\mathrm{J}_{\mathrm{QV}}-\mathrm{J}_{\mathrm{Q} \theta} \mathrm{J}_{\mathrm{P} \theta^{-1}} \mathrm{JPV}\right)
$$

$\mathrm{J}_{\mathrm{R}}$ is called the reduced Jacobian matrix of the system.

\subsection{Modes of Voltage Instability}

Voltage Stability characteristics of the system have been identified by computing the Eigen values and Eigen vectors.

$$
\begin{aligned}
& \text { Let } \quad \mathrm{J}_{\mathrm{R}}=\xi \wedge \eta \\
& \text { where, } \quad \xi=\text { right eigenvector matrix of JR } \\
& \eta=\text { left eigenvector matrix of JR } \\
& \wedge=\text { diagonal eigenvalue matrix of JR and } \\
& \mathrm{J}_{\mathrm{R}^{-1}}=\xi_{\wedge}{ }^{-1} \eta
\end{aligned}
$$

From (5) and (8), we have

$$
\begin{aligned}
\Delta V & =\xi_{\wedge}{ }^{-1} \eta \Delta \mathrm{Q} \\
\text { or, } \Delta & \Delta \mathrm{V}=\sum_{\mathrm{I}} \frac{\xi_{\mathrm{i}} \eta_{\mathrm{i}}}{\lambda_{\mathrm{i}}} \Delta \mathrm{Q}
\end{aligned}
$$

where $\xi_{\mathrm{i}}$ is the ith column right eigenvector and $\eta$ the ith row left eigenvector of JR.

$\lambda_{\mathrm{i}}$ is the ith Eigen value of JR.

The $i^{\text {th }}$ modal reactive power variation is

$$
\Delta \mathrm{Q}_{\mathrm{mi}}=\mathrm{K}_{\mathrm{i}} \xi_{\mathrm{i}}
$$

where,

$$
\mathrm{K}_{\mathrm{i}}=\sum_{\mathrm{j}} \xi_{\mathrm{ij}}-1
$$

$\xi_{\mathrm{ji}}$ is the jth element of $\xi_{\mathrm{i}}$

The corresponding ith modal voltage variation is

$$
\Delta \mathrm{V}_{\mathrm{mi}}=\left[1 / \lambda_{\mathrm{i}}\right] \Delta \mathrm{Q}_{\mathrm{mi}}
$$

If $\left|\lambda_{i}\right|=0$ then the ith modal voltage will collapse .

In (10), let $\Delta \mathrm{Q}=\mathrm{ek}$ where ek has all its elements zero except the kth one being 1 . Then,

$\eta_{1 \mathrm{k}} \quad \mathrm{k}^{\text {th }}$ element of $\eta_{1}$

$$
\Delta \mathrm{V}=\sum_{\mathrm{i}} \frac{\eta_{1 \mathrm{k}} \xi_{1}}{\lambda_{1}}
$$

$\mathrm{V}-\mathrm{Q}$ sensitivity at bus $\mathrm{k}$

$$
\frac{\partial \mathrm{V}_{\mathrm{K}}}{\partial \mathrm{Q}_{\mathrm{K}}}=\sum_{\mathrm{i}} \frac{\eta_{1 \mathrm{k}} \xi_{1}}{\lambda_{1}}=\sum_{\mathrm{i}} \frac{\mathrm{P}_{\mathrm{ki}}}{\lambda_{1}}
$$




\section{Problem Formulation}

The objectives of the reactive power dispatch problem is to minimize the system real power loss and maximize the static voltage stability margins (SVSM).

\subsection{Minimization of Real Power Loss}

Minimization of the real power loss (Ploss) in transmission lines is mathematically stated as follows.

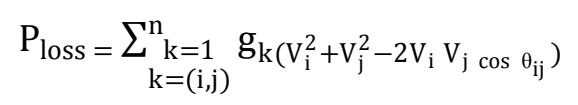

where $n$ is the number of transmission lines, $\mathrm{g}_{\mathrm{k}}$ is the conductance of branch $\mathrm{k}, \mathrm{V}_{\mathrm{i}}$ and $\mathrm{V}_{\mathrm{j}}$ are voltage magnitude at bus $i$ and bus $j$, and $\theta_{\mathrm{ij}}$ is the voltage angle difference between bus $i$ and bus j.

\subsection{Minimization of Voltage Deviation}

Minimization of the voltage deviation magnitudes (VD) at load buses is mathematically stated as follows:

$$
\text { Minimize } \mathrm{VD}=\sum_{\mathrm{k}=1}^{\mathrm{nl}}\left|\mathrm{V}_{\mathrm{k}}-1.0\right|
$$

where $n l$ is the number of load busses and $\mathrm{V}_{\mathrm{k}}$ is the voltage magnitude at bus $\mathrm{k}$.

\subsection{System Constraints}

Objective functions are subjected to these constraints shown below.

Load flow equality constraints:

$$
\begin{aligned}
& P_{G i}-P_{D i}-V_{i} \sum_{j=1}^{n b} V_{j}\left[\begin{array}{cc}
G_{i j} & \cos \theta_{i j} \\
+B_{i j} & \sin \theta_{i j}
\end{array}\right]=0, i=1,2 \ldots, n b \\
& Q_{G i}-Q_{D i}-V_{i} \sum_{j=1}^{n b} V_{j}\left[\begin{array}{cc}
G_{i j} & \sin \theta_{i j} \\
+B_{i j} & \cos \theta_{i j}
\end{array}\right]=0, i=1,2 \ldots, n b
\end{aligned}
$$

where, $\mathrm{nb}$ is the number of buses, $\mathrm{P}_{\mathrm{G}}$ and $\mathrm{Q}_{\mathrm{G}}$ are the real and reactive power of the generator, $\mathrm{P}_{\mathrm{D}}$ and $Q_{D}$ are the real and reactive load of the generator, and $\mathrm{G}_{\mathrm{ij}}$ and $\mathrm{B}_{\mathrm{ij}}$ are the mutual conductance and susceptance between bus $i$ and bus $j$.

Generator bus voltage (VGi) inequality constraint:

$$
\mathrm{V}_{\mathrm{Gi}}^{\min } \leq \mathrm{V}_{\mathrm{Gi}} \leq \mathrm{V}_{\mathrm{Gi}}^{\max }, \mathrm{i} \in \mathrm{ng}
$$

Load bus voltage (VLi) inequality constraint: 


$$
\mathrm{V}_{\mathrm{Li}}^{\min } \leq \mathrm{V}_{\mathrm{Li}} \leq \mathrm{V}_{\mathrm{Li}}^{\max }, \mathrm{i} \in \mathrm{nl}
$$

Switchable reactive power compensations (QCi) inequality constraint:

$$
\mathrm{Q}_{\mathrm{Ci}}^{\min } \leq \mathrm{Q}_{\mathrm{Ci}} \leq \mathrm{Q}_{\mathrm{Ci}}^{\max }, \mathrm{i} \in \mathrm{nc}
$$

Reactive power generation (QGi) inequality constraint:

$$
\mathrm{Q}_{\mathrm{Gi}}^{\min } \leq \mathrm{Q}_{\mathrm{Gi}} \leq \mathrm{Q}_{\mathrm{Gi}}^{\max }, \mathrm{i} \in \mathrm{ng}
$$

Transformers tap setting (Ti) inequality constraint:

$$
\mathrm{T}_{\mathrm{i}}^{\min } \leq \mathrm{T}_{\mathrm{i}} \leq \mathrm{T}_{\mathrm{i}}^{\max }, \mathrm{i} \in \mathrm{nt}
$$

Transmission line flow (SLi) inequality constraint:

$$
\mathrm{S}_{\mathrm{Li}}^{\min } \leq \mathrm{S}_{\mathrm{Li}}^{\max }, \mathrm{i} \in \mathrm{nl}
$$

where, $n c, n g$ and $n t$ are numbers of the switchable reactive power sources, generators and transformers

\section{Eagle Strategy}

Eagle strategy (ES) is a metaheuristics approach for optimization, developed in 2010 by Xin-She Yang and Suash Deb [15]. It uses a mixture of crude global search and intensive local search. In essence, the strategy first explores the search space globally using a Levy flight random walk, if it finds a promising solution, then a concentrated local search is employed using a well-organized local optimizer such as hill-climbing, differential evolution and algorithms. Then, the two-stage process starts again with new comprehensive exploration followed by a local search in a new area. In this approach mainly $p_{e}$ and which controls the switch between local and global search. Essentially, ES makes the global search in the $n$-dimensional space with Levy flights; if any probable solution is found, an intensive local optimizer is put to use for local search such as differential evolution, particle swarm optimization algorithm, and artificial bee colony that these have local search capability. Then the procedure starts again with new global search in the new area.

Levy distribution is given as follows:

$$
L \sim \frac{\lambda \Gamma(\lambda \sin (\Pi \lambda / 2))}{\Pi} \frac{1}{s^{1+\lambda}},\left(s \gg s_{0}>0\right)
$$

Here, $\Gamma(\lambda)$ is the standard gamma function, and this distribution is valid for large steps $s>0$.

\section{Eagle strategy algorithm}

[Step1]. Objective functions $\mathrm{f}(\mathrm{x})$

[Step2]. Initialization and random initial presumption $\mathrm{x}^{\mathrm{t}=0}$

[Step3]. while (stop criterion) 
[Step4]. Global exploration by randomization

[Step5]. Evaluate the objective

[Step6]. If $\mathrm{p}_{\mathrm{e}}<$ rand, switch to a local search

[Step7]. Intensive local search around a capable solution through an well-organized optimizer

[Step8]. if (a better solution is found)

[Step9]. Update the current best

End

End

[Step10]. Update $t=t+1$

End

[Step11]. Post -route the results and revelation.

\section{Particle Swarm Optimization Algorithm}

The particle swarm optimization algorithm (PSO) [16] conducts searches using a population of particles which correspond to individuals in GAs. The population of particles is randomly generated initially. Each particle represents a potential solution and has a position represented by a position vector $\overrightarrow{x_{i}}$. A swarm of particles moves through the problem space, with the moving velocity of each particle represented by a position vector $\overrightarrow{v_{i}}$ At each time step, a function $f_{i}$ representing a quality measure is calculated by using $\overrightarrow{x_{i}}$ as input. Each particle keeps track of its own best position, which is associated with the best fitness it has achieved so far in a vector $\overrightarrow{p_{i}}$. Furthermore, the best position among all the particles obtained so far in the population is kept track of as $\overrightarrow{p_{g}}$. At each time step $\boldsymbol{\tau}$, by using the individual best position, $\overrightarrow{p_{i}}(\boldsymbol{\tau})$ and global best position, $\overrightarrow{p_{g}}(\boldsymbol{\tau})$ a new velocity for particle $\mathrm{i}$ is updated by

$\overrightarrow{v_{i}}(\tau+1)=\omega \overrightarrow{v_{i}}(\tau)+c_{1} \emptyset_{1}\left(\overrightarrow{p_{i}}(\tau)-\overrightarrow{x_{i}}(\tau)\right)+c_{2} \emptyset_{2}\left(\overrightarrow{p_{g}}(\tau)-\overrightarrow{x_{i}}(\tau)\right)$

where $c_{1}$ and $c_{2}$ are acceleration constants and $\emptyset_{1} \& \emptyset_{2}$ are uniformly distributed random numbers in $[0,1]$. The term $\overrightarrow{v_{i}}$ is limited to its bounds. If the velocity violates this limit, it is set to its proper limit.

$\omega$ is the inertia weight factor and in general, it is set according to the following equation:

$$
\omega=\omega_{\max }-\frac{\omega_{\max }-\omega_{\min }}{T} \cdot \boldsymbol{\tau}
$$

where $\omega_{\max }$ and $\omega_{\min }$ is maximum and minimum value of the weighting factor respectively. $T$ is the maximum number of iterations and $\tau$ is the current iteration number. Based on the updated velocities, each particle changes its position according to the following:

$$
\overrightarrow{x_{i}}(\tau+1)=\overrightarrow{x_{i}}(\tau)+h(\tau) \overrightarrow{v_{i}}(\tau+1)
$$

where 


$$
h(\tau)=h_{\max }-\frac{\left(h_{\max }-h_{0}\right) \cdot \tau}{T}
$$

$h_{\text {max }}$ and $h_{0}$ are positive constants.

According to (25) and (27), the populations of particles tend to cluster together with each particle moving in a random direction.

Particle swarm optimization algorithm

Load objective function $(x)$

Generate the initial population and velocity of $n$ particles

Find global best (at $k=0$ )

While $(\|\operatorname{minimum}(k+1)-\operatorname{minimum} f(k)\| \leq$ tolerance or

$k>$ max number of iterations)

Calculate new velocity and position of each particle

Evaluate the new fitness

If $\left(x_{i}(k+1)\right)<f\left(\right.$ best $\left._{i}(k)\right)$

$p \operatorname{best}(k+1)=x_{i}(k+1)$

Update global best

Update weight

$k=k+1$

\section{End}

\section{Hybridization of Eagle Strategy with Particle Swarm Optimization Algorithm}

PSO is applied to the local with Levy walks can be used in the global search. The proposed method is a population-based algorithm. We used the parameters of PSO used in most applications, where $c 1=c 2=2, w \min =0.4$, and $w \max =0.9$; then we set $\lambda=2$. We used 500 iterations for benchmark functions and 100 iterations for reactive power optimization problem. There are two important situations, $\Gamma \rightarrow \infty$ and $\Gamma \rightarrow 0$. If $\Gamma \rightarrow \infty$, then the velocity of particles cannot be decreased particles are far from one another. If $\Gamma \rightarrow 0$, then the particles are short sighted, so particles will be trapped in a confined space and velocity of this particles can be very small. There are several stopping criteria given in the literature: a fixed number of generations, the number of iterations since the last change of the best solution being greater than a specified number, the number of iterations reaching maximum number, a located string with a certain value, and no change in the average fitness after some generations. In this paper, the stopping criteria are chosen as the maximum number of iterations and the tolerance value for fitness where \|\| minimum $(k+1)-\operatorname{minimum} f(k)\|\| \leq$ tolerance.

Step 1. Load function and its parameters

Step 2. Produce preliminary population randomly

Step 3. While \|\| $\operatorname{minimum}(k+1)-\operatorname{minimum} f(k)\|\| \leq$ tolerance or 
$k>\max$ number of iterations,

Performing arbitrary global exploration using Levy Flight $x^{k^{+1}}=x k+\alpha(s, \lambda)$, ( $\lambda=1.5, \alpha=1$, and step length $s$ set as $s=5$ )

Then, find a capable solution

Step 4. Establish a arbitrary number. Set switching parameter $p$ for controlling between global search and local search. (We set $p=0.2$ )

If $p<$ rand

Switch to local search stage (go to Step 5)

Else

Switch to global search stage (go to Step 6)

Step 5. In exhaustive local search stage, search around a capable solution,

Compute new velocity and position of each particle

Then appraise new fitness (Use the objective function based on NewtonRaphson power flow for reactive power optimization problem)

If $\left(x_{i}(k+1)\right)<f\left(p \operatorname{best}_{i}(k)\right)$

$p \operatorname{best}(k+1)=x_{i}(k+1)$

Step 6. Update, $k=k+1$

Step 7. End criterion,

Maximum number of iterations or a given tolerance (tolerance set as $1.0000 e$ -9 for reactive power optimization problem)

Step 8. If any criterion is provided, then stop the algorithm else go to Step 3.

\section{Simulation Results}

The efficiency of the proposed hybridization of Eagle Strategy with Particle Swarm Optimization (EPSO) is demonstrated by testing it on standard IEEE-30 bus system. The IEEE-30 bus system has 6 generator buses, 24 load buses and 41 transmission lines of which four branches are (6-9), $(6-10),(4-12)$ and (28-27) - are with the tap setting transformers. The lower voltage magnitude limits at all buses are 0.95 p.u. and the upper limits are 1.1 for all the PV buses and 1.05 p.u. for all the PQ buses and the reference bus. The simulation results have been presented in Tables 1,2, $3 \& 4$. And in the Table 5 shows the proposed algorithm powerfully reduces the real power losses when compared to other given algorithms. The optimal values of the control variables along with the minimum loss obtained are given in Table 1. Corresponding to this control variable setting, it was found that there are no limit violations in any of the state variables. 
Table 1. Results of EPSO - ORPD optimal control variables

\begin{tabular}{|c|c|}
\hline Control variables & Variable setting \\
\hline V1 & 1.043 \\
V2 & 1.044 \\
V5 & 1.046 \\
V8 & 1.030 \\
V11 & 1.003 \\
V13 & 1.030 \\
T11 & 1.00 \\
T12 & 1.00 \\
T15 & 1.01 \\
T36 & 1.01 \\
Qc10 & 2 \\
Qc12 & 3 \\
Qc15 & 2 \\
Qc17 & 0 \\
Qc20 & 2 \\
Qc23 & 2 \\
Qc24 & 3 \\
Qc29 & 2 \\
Real power loss & 4.2792 \\
SVSM & 0.2484 \\
\hline
\end{tabular}

Optimal Reactive Power Dispatch problem together with voltage stability constraint problem was handled in this case as a multi-objective optimization problem where both power loss and maximum voltage stability margin of the system were optimized simultaneously. Table 2 indicates the optimal values of these control variables. Also it is found that there are no limit violations of the state variables. It indicates the voltage stability index has increased from 0.2484 to 0.2496 , an advance in the system voltage stability. To determine the voltage security of the system, contingency analysis was conducted using the control variable setting obtained in case 1 and case 2 . The Eigen values equivalents to the four critical contingencies are given in Table 3 . From this result it is observed that the Eigen value has been improved considerably for all contingencies in the second case. 
Table 2. Results of EPSO -Voltage Stability Control Reactive Power Dispatch Optimal Control Variables

\begin{tabular}{|c|c|}
\hline Control Variables & Variable Setting \\
\hline V1 & 1.048 \\
V2 & 1.049 \\
V5 & 1.047 \\
V8 & 1.030 \\
V11 & 1.004 \\
V13 & 1.031 \\
T11 & 0.090 \\
T12 & 0.090 \\
T15 & 0.090 \\
T36 & 0.090 \\
Qc10 & 3 \\
Qc12 & 2 \\
Qc15 & 2 \\
Qc17 & 3 \\
Qc20 & 0 \\
Qc23 & 2 \\
Qc24 & 2 \\
Qc29 & 3 \\
Real power loss & 4.9896 \\
SVSM & 0.2496 \\
\hline
\end{tabular}

Table 3. Voltage Stability under Contingency State

\begin{tabular}{|c|c|c|c|}
\hline S1.No & Contingency & ORPD Setting & VSCRPD Setting \\
\hline 1 & $28-27$ & 0.1419 & 0.1434 \\
\hline 2 & $4-12$ & 0.1642 & 0.1650 \\
\hline 3 & $1-3$ & 0.1761 & 0.1772 \\
\hline 4 & $2-4$ & 0.2022 & 0.2043 \\
\hline
\end{tabular}


32 Real Power Loss Minimization and Voltage Stability Enhancement by Hybridization of Eagle Strategy ...

Table 4. Limit Violation Checking Of State Variables

\begin{tabular}{|c|c|c|c|c|}
\hline \multirow{2}{*}{ State variables } & \multicolumn{2}{|c|}{ limits } & \multirow{2}{*}{ ORPD } & \multirow{2}{*}{ VSCRPD } \\
\hline & Lower & upper & & \\
\hline Q1 & -20 & 152 & 1.3422 & -1.3269 \\
\hline Q2 & -20 & 61 & 8.9900 & 9.8232 \\
\hline Q5 & -15 & 49.92 & 25.920 & 26.001 \\
\hline Q8 & -10 & 63.52 & 38.8200 & 40.802 \\
\hline Q11 & -15 & 42 & 2.9300 & 5.002 \\
\hline Q13 & -15 & 48 & 8.1025 & 6.033 \\
\hline V3 & 0.95 & 1.05 & 1.0372 & 1.0392 \\
\hline V4 & 0.95 & 1.05 & 1.0307 & 1.0328 \\
\hline V6 & 0.95 & 1.05 & 1.0282 & 1.0298 \\
\hline V7 & 0.95 & 1.05 & 1.0101 & 1.0152 \\
\hline V9 & 0.95 & 1.05 & 1.0462 & 1.0412 \\
\hline V10 & 0.95 & 1.05 & 1.0482 & 1.0498 \\
\hline V12 & 0.95 & 1.05 & 1.0400 & 1.0466 \\
\hline V14 & 0.95 & 1.05 & 1.0474 & 1.0443 \\
\hline V15 & 0.95 & 1.05 & 1.0457 & 1.0413 \\
\hline V16 & 0.95 & 1.05 & 1.0426 & 1.0405 \\
\hline V17 & 0.95 & 1.05 & 1.0382 & 1.0396 \\
\hline V18 & 0.95 & 1.05 & 1.0392 & 1.0400 \\
\hline V19 & 0.95 & 1.05 & 1.0381 & 1.0394 \\
\hline V20 & 0.95 & 1.05 & 1.0112 & 1.0194 \\
\hline V21 & 0.95 & 1.05 & 1.0435 & 1.0243 \\
\hline V22 & 0.95 & 1.05 & 1.0448 & 1.0396 \\
\hline V23 & 0.95 & 1.05 & 1.0472 & 1.0372 \\
\hline V24 & 0.95 & 1.05 & 1.0484 & 1.0372 \\
\hline V25 & 0.95 & 1.05 & 1.0142 & 1.0192 \\
\hline V26 & 0.95 & 1.05 & 1.0494 & 1.0422 \\
\hline V27 & 0.95 & 1.05 & 1.0472 & 1.0452 \\
\hline V28 & 0.95 & 1.05 & 1.0243 & 1.0283 \\
\hline V29 & 0.95 & 1.05 & 1.0439 & 1.0419 \\
\hline V30 & 0.95 & 1.05 & 1.0418 & 1.0397 \\
\hline
\end{tabular}


Table 5. Comparison of Real Power Loss

\begin{tabular}{|c|c|}
\hline Method & Minimum loss \\
\hline Evolutionary programming [14] & 5.0159 \\
\hline Genetic algorithm [9] & 4.665 \\
\hline Real coded GA with Lindex as SVSM [8] & 4.568 \\
\hline Real coded genetic algorithm [5] & 4.5015 \\
\hline Proposed EPSO method & 4.2792 \\
\hline
\end{tabular}

\section{Conclusion}

In this paper, hybridization of Eagle Strategy with Particle Swarm Optimization (EPSO) has been applied to solve optimal reactive power dispatch problem. Different objective functions have been utilized to diminish real power loss and the voltage profile has been improved. Projected hybridized Eagle Strategy with Particle Swarm Optimization (EPSO) algorithm has been tested on the standard IEEE 30-bus power system \& simulation results indicate the effectiveness and robustness of the projected hybridized Eagle Strategy with Particle Swarm Optimization (EPSO) in solving optimal reactive power dispatch problem.

\section{References}

[1] Abido MA and Bakhashwain JM (2003), A novel multi objective evolutionary algorithm for optimal reactive power dispatch problem, in proc. Electronics, Circuits and Systems conf., 3 : 1054-1057.

[2] Abdullah WNW, Saibon H, Zain AM and Lo KL (1998), Genetic Algorithm for Optimal Reactive Power Dispatch, in proc. Energy Management and Power Delivery conf., 1 : 160164.

[3] Abido MA (2002), Optimal Power Flow Using Particle Swarm Optimization, Electrical Power and Energy Systems, $24: 563-571$.

[4] Abou El Ela AA, Abido MA and Spea SR (2011), Differential Evolution Algorithm for Optimal Reactive Power Dispatch, Electric Power Systems Research, 81:458-464.

[5] Aruna JP and Devaraj D (2010), Optimal Reactive Power Dispatch for Voltage Stability Enhancement Using Real Coded Genetic Algorithm, International Journal of Computer and Electrical Engineering, 2 (4) : 1793-8163.

[6] Canizares CA, de Souza ACZ and Quintana VH (1996), Comparison of performance indices for detection of proximity to voltage collapse, IEEE Trans. on Power Systems, 11(3) : 14411450.

[7] Deeb NI and Shahidehpour SM (1988), An Efficient Technique for Reactive Power Dispatch Using a Revised Linear Programming Approach, Electric Power System Research, 15 : 121134. 
34 Real Power Loss Minimization and Voltage Stability Enhancement by Hybridization of Eagle Strategy ...

[8] Devaraj D (2007), Improved genetic algorithm for multi - objective reactive power dispatch problem, European Transactions on electrical power, 17: 569-581.

[9] Durairaj S, Devaraj D and Kannan PS (2006), Genetic algorithm applications to optimal reactive power dispatch with voltage stability enhancement", IE(I) Journal-EL, 87 : 111119.

[10] Grudinin N (1998), Reactive Power Optimization Using Successive Quadratic Programming Method, IEEE Trans. on Power Systems, 3 : 1219-1225.

[11] Granville S (1994), Optimal Reactive Dispatch Trough Interior Point Methods,IEEE Trans. on Power Systems, 9 : 136-146.

[12] Lee KY, Park YM and Ortiz JL (1984), Fuel-cost minimization for both real and reactivepower dispatches, in proc Generation, Transmission and Distribution conf, 131 : 85-93.

[13] Miranda VNF (2002), EPSO-Evolutionary Particle Swarm Optimization, A New Algorithm with Applications in Power Systems, in Proc of Transmission and Distribution conf, 2 : 745750.

[14] Wu QH and Ma JT (1995), Power system optimal reactive power dispatch using evolutionary programming, IEEE Transactions on power systems; 10(3): 1243-1248.

[15] Yang XS and Deb S (2010), Eagle strategy using Levy walk and firefly algorithm for stochastic optimization, in: Nature Inspired Cooperative Strategies for Optimization (NICSO 2010) (Eds. J. R. Gonzalez et al.), SCI, $284: 101-111$.

[16] Zhang W and Liu YT (2008), Multi-objective reactive power and voltage control based on fuzzy optimization strategy and fuzzy adaptive particle swarm, International Journal of Electrical Power and Energy Systems, 30(9) : 525-532. 